\title{
Normal echocardiographic mitral and aortic valve thickness in children
}

\author{
Rachel H Webb, ${ }^{1,2}$ Nicola Culliford-Semmens, ${ }^{1}$ Karishma Sidhu, ${ }^{3}$ Nigel J Wilson ${ }^{1}$
}

\begin{abstract}
${ }^{1}$ Green Lane Paediatric and Congenital Cardiac Services, Starship Children's Hospital, Auckland, New Zealand ${ }^{2}$ Paediatric Infectious Diseases, Starship Children's Hospital, Auckland, New Zealand ${ }^{3}$ Green Lane Cardiovascular Services, Auckland City Hospital, Auckland, New Zealand
\end{abstract}

\section{Correspondence to} Dr Rachel H Webb, Paediatric Infectious Diseases, Starship Children's Hospital, Private Bag 92 024, Auckland, New Zealand; rwebb@adhb.govt.nz

Received 15 December 2016 Revised 14 February 2017 Accepted 15 February 2017

\begin{abstract}
Objective We aimed to define the normal range of aortic and mitral valve thickness in healthy schoolchildren from a high prevalence rheumatic heart disease (RHD) region, using a standardised protocol for imaging and measurement.

Methods Measurements were performed in 288 children without RHD. Anterior mitral valve leaflet (AMVL) thickness measurements were performed at the midpoint and tip of the leaflet in the parasternal long axis (PSLA) in diastole, when the AMVL was approximately parallel to the ventricular septum. Thickness of the aortic valve was measured from PSLA imaging in systole when the leaflets were at maximum excursion. The right coronary and non-coronary closure lines of the aortic valve were measured in diastole in parasternal short axis (PSSA) imaging. Results were compared with 51 children with RHD classified by World Heart Federation diagnostic criteria.

Results In normal children, median AMVL tip thickness was $2.0 \mathrm{~mm}$ (IQR 1.7-2.4) and median AMVL midpoint thickness $2.0 \mathrm{~mm}$ (IQR 1.7-2.4). The median aortic valve thickness was $1.5 \mathrm{~mm}$ (IQR 1.3-1.6) in the PSLA view and $1.4 \mathrm{~mm}$ (IQR 1.2-1.6) in the PSSA view. The interclass correlation coefficient for the AMVL tip was 0.85 (0.71 to 0.92$)$ and for the AMVL midpoint was 0.77 (0.54 to 0.87 ).

Conclusions We have described a standardised method for mitral and aortic valve measurement in children which is objective and reproducible. Normal ranges of left heart valve thickness in a high prevalence RHD population are established. These results provide a reference range for school-age children in high prevalence RHD regions undergoing echocardiographic screening.
\end{abstract}

\section{INTRODUCTION}

Thickening of both the mitral ${ }^{1-4}$ and aortic valve $^{4-6}$ leaflets are features of established rheumatic heart disease (RHD). The 2012 World Heart Federation (WHF) consensus diagnostic criteria for RHD include thickening as one of the morphological criteria. ${ }^{4}$ Increased leaflet thickness of mitral valve leaflets has also been observed in children with acute rheumatic fever (ARF). ${ }^{7}$

In school-age children, echocardiographic screening studies for RHD have used valve thickening as one of the morphological diagnostic criteria ${ }^{8-13}$ and yet the normal range in this population has not been established. There are also limited data about the echocardiographic thickness of mitral valves in children with $\mathrm{ARF}^{7}{ }^{14}$ and RHD. ${ }^{14}{ }^{15}$ Some authors have relied on subjective evaluation, and other more recent RHD echo studies have used an arbitrary cut-off of $3 \mathrm{~mm}$ for anterior mitral valve leaflet (AMVL) tip.

\section{AIMS}

The primary aim of this study was to define the normal range of aortic and mitral valve thickness in healthy school-age children from a high prevalence RHD region using a standardised objective protocol for imaging and measurement. A secondary aim was to establish whether mitral and aortic valve thickness differs in children with and without RHD.

\section{METHODS}

\section{Study design and participants}

Population-based echocardiographic screening was conducted in children aged 10-13 years in South Auckland, New Zealand, a region with a high incidence of ARF/RHD. ${ }^{12}{ }^{16}$ A consecutive series of 288 echocardiograms from children with no evidence of RHD or congenital valvular abnormality formed the study group to establish the normal range of valve thickness. Those with no mitral regurgitation or a closing volume of mitral regurgitation $^{17}$ and with normal morphological appearances were included, but those with true physiological regurgitation ${ }^{17}$ were excluded from analysis in case they had borderline RHD. Valve thickness measurements were also obtained from 51 subjects with RHD. These echocardiograms were obtained during the same previously published population-based RHD prevalence study. $^{12}{ }^{18}$ The RHD classification by the WHF criteria was performed in a re-analysis of cases by a panel of three cardiologists. This was necessary as the WHF criteria had not been published at the time of the original study. ${ }^{18}$

Two children who met diagnostic criteria for $\mathrm{ARF}$ at the time of screening were excluded from the analysis of the RHD group, as oedema and inflammation in the acute phase of ARF could lead to an overestimate of valve thickness.

Ethical approval was obtained from the New Zealand Regional Ethics Committee: reference NTY/06/12/139.

\section{Echocardiographic procedures}

Echocardiography was performed using a standardised protocol. Vivid e (GE Healthcare) portable cardiac ultrasound machines were used. A $2-3 \mathrm{mHz}$ variable frequency probe was used. Twodimensional Doppler images were obtained in parasternal and apical four-chamber views. Transducer gain settings were optimised individually by experienced cardiac sonographers, as the large variation in body habitus precluded the use of standardised 
gain settings. The study protocol stipulated that Harmonics should be turned off for two-dimensional (2D) imaging, unless image acquisition was suboptimal.

\section{Valve thickness measurements}

Measurements were performed using the software package of the ultrasound machine rather than measured off-line. Measurements were made by two separate observers who were blinded to each other's measurements. Individual measurements were made three times from optimal still frames of cine-loops.
Measurements were made in millimetres to an accuracy of two decimal places as per machine settings then averaged to millimetres and one decimal point.

Measurements of the AMVL thickness were performed at the midpoint and the tip of the leaflet in the parasternal long axis (PSLA) imaging in diastole, when the AMVL was approximately parallel to the ventricular septum (figure 1A,B). This was usually the still frame of maximal diastolic excursion of the AMVL. Measurement of the AMVL midpoint was also performed in systole from apical four-chamber imaging (figure $1 \mathrm{C}$ ).
Figure 1 Two-dimensional echocardiography image of each imaging view, and a corresponding cartoon diagram showing the method for each valve leaflet measurement. (A) Parasternal long axis in late diastole. (B) Parasternal long axis in late diastole. (C) Apical four-chamber view. (D) Parasternal long axis in systole. (E) Parasternal short axis in diastole. Ao, aorta; LA, left atrium; $L V$, left ventricle; $R V$, right ventricle; $\mathrm{PA}$, pulmonary artery.
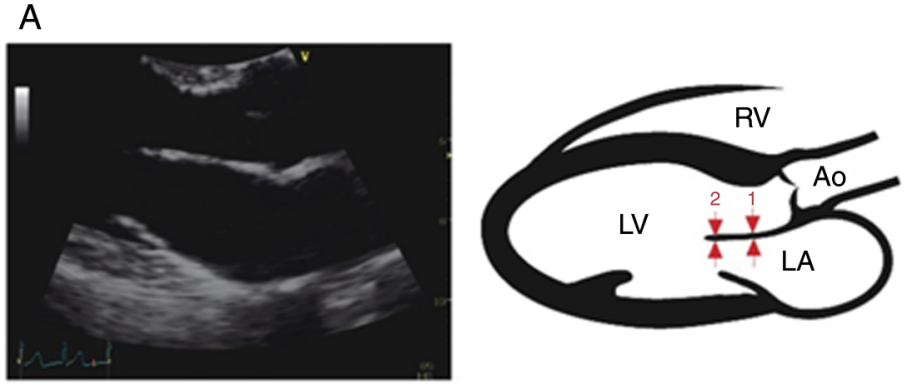

1. Measurement for anterior mitral valve leaflet midpoint

2. Measurement for anterior mitral valve leaflet tip

B
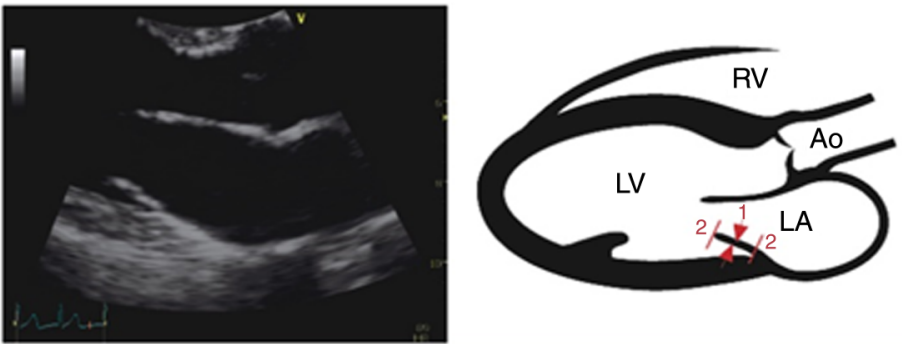

1. Measurement for posterior mitral valve leaflet midpoint

2. Measurement for posterior mitral valve leaflet length

C
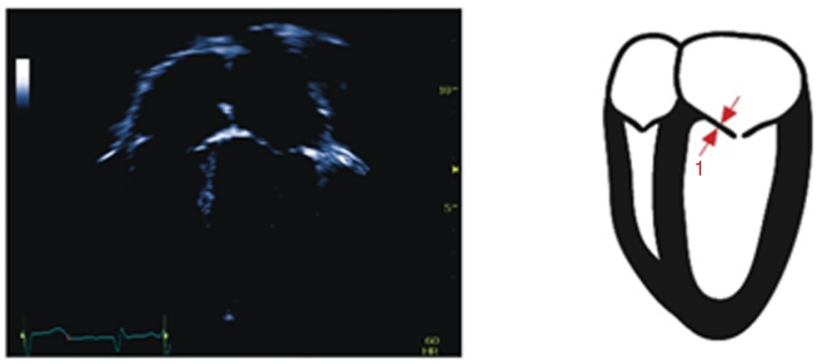

1. Measurement for anterior mitral valve leaflet midpoint

D
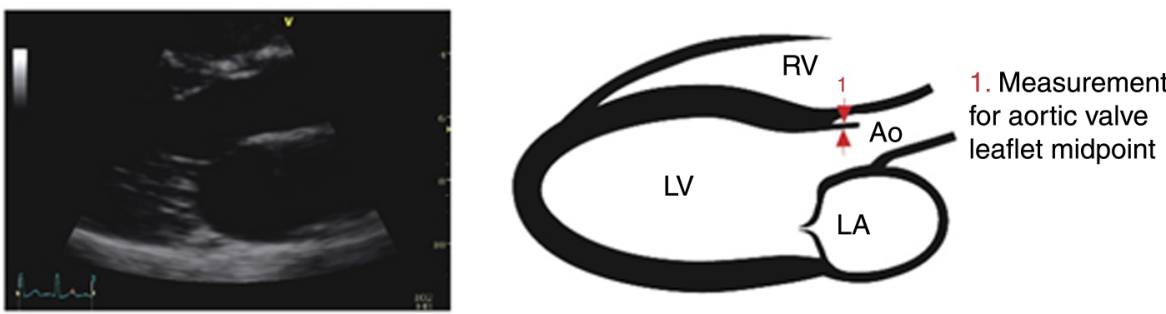

E
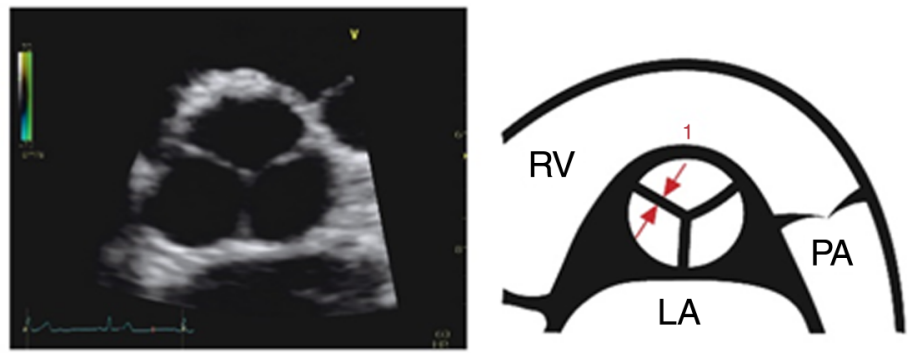

Measurement for the right coronary cusp and non-coronary cusp 
Measurements of the posterior mitral valve leaflet (PMVL) thickness (figure 1C) and length were measured in diastole in a still frame where there was clear separation of the PMVL from other posterior annulus tissues.

The thickness of the aortic valve was measured from PSLA imaging in systole when the leaflets were at maximum excursion (figure 1D). The right coronary and non-coronary closure lines of the aortic valve were also measured in diastole in parasternal short axis (PSSA) imaging (figure 1E).

\section{Statistical analyses}

Thickness and length measurements of aortic and mitral valves were summarised at median with IQRs. The Wilcoxon rank-sum test was used to assess the difference in these measurements between normal and individuals with RHD. Negative predictive valve (NPV) and positive predictive value (PPV) were calculated using standard formulae, for aortic and mitral valve thickness. The intraclass correlation coefficient was calculated to assess the interobserver agreement between two observers, and the intraobserver agreement for both of the observers, for a subgroup of normal individuals only. Statistical analyses were performed using the statistical package SAS V.9.3 (SAS Institute, Cary, North Carolina, USA). All p values resulted from twosided tests.

\section{RESULTS}

\section{Normals}

The normal valve measurements in 288 normal children and children aged 10-13 years are presented in table 1 . Figure 2 demonstrates normal mitral valve appearances. In the PSLA view, the AMVL tip thickness was $2.0 \mathrm{~mm}$ (IQR 1.7-2.4) and AMVL midpoint thickness $2.0 \mathrm{~mm}$ (IQR 1.7-2.4). There was no statistically significant difference in measurement whether the leaflet was measured at the tip or midpoint, or whether it was measured in the parasternal or apical view $(p=0.9)$.

The uppermost measurement for both AMVL tip and midpoint in normal subjects was $2.9 \mathrm{~mm}$.

The midpoint of the PMVL measured $2.2 \mathrm{~mm}$ (IQR 1.9-2.5). This was significantly thicker than the midpoint of the AMVL $(\mathrm{p}<0.0001)$.

The median thickness of the normal aortic valve was $1.5 \mathrm{~mm}$ (IQR 1.3-1.6) in the PSLA view and $1.4 \mathrm{~mm}$ (IQR 1.2-1.6) in the PSSA view. There was no significant difference between these two views $(p=0.4)$.

\section{Rheumatic heart disease}

There were 51 RHD cases altogether. Thirteen cases had definite RHD and 38 cases had borderline RHD. Among the 38 borderline RHD cases, 21 had mitral regurgitation, 12 had aortic regurgitation and 5 had morphological changes. None had mitral stenosis.

Valve measurements for the children with RHD are presented in table 1. The AMVL tip measured $2.6 \mathrm{~mm}$ (IQR 2.3-3.2) and midpoint $2.8 \mathrm{~mm}$ (IQR 2.5-3.2) in the PSLA view in this group. Valve thickness of the aortic valve in children with RHD measured $1.9 \mathrm{~mm}$ (IQR 1.8-2.1) in the PSLA view and $1.9 \mathrm{~mm}$ (IQR 1.7-2.1) in the PSSA view.

A comparison of mitral and aortic valve thickness between the normal group and RHD group is presented in table 1 . The AMVL, PMVL and aortic valve leaflet were all thicker in children with RHD than in normal children in all imaging views.

\section{Positive and negative predictive values}

The NPV $\leq 3 \mathrm{~mm}$ for mitral thicknesses is shown in table 2, with the best predictor being the AMVL measurement in the PSLA view.

The NPV cut-off of $\leq 2 \mathrm{~mm}$ thickness for aortic valve thickness is shown in table 2. PPVs for respective mitral and aortic cut-offs are shown in table 3.

\section{Interobserver and intraobserver variability results (normal)}

Interobserver agreement: the interclass correlation coefficient for the AMVL tip was 0.85 (0.71 to 0.92) and for the AMVL midpoint was 0.77 (0.54 to 0.87 ).

Intraobserver agreement for AMVL measurements is shown in table 4.

\section{DISCUSSION}

This study describes an objective standardised methodology for valve thickness measurements from echocardiograms obtained from a RHD school screening programme in a high incidence ARF region. The range of normal echocardiographic valve thickness measurements in a population of children aged 1013 years provides a reference dataset suitable for use in RHD echocardiographic screening programmes in children.

\section{Mitral valve}

The results showed that the AMVL measured $2.0 \mathrm{~mm}$ (IQR 1.7-2.4) in a normal population of children aged 10-13 years. There was no difference whether the measurement was taken at the tip or the midpoint of the leaflet, or whether the measurement was performed in parasternal or apical imaging (table 1). The distance from the transducer to the mitral valve is closer in parasternal than apical imaging so intuitively this may be the preferable method in subjects with poor echocardiographic windows. The posterior leaflet thickness was similar, but the observers noted that it could be difficult to make measurements perpendicular to the leaflet in parasternal imaging.

Table 1 Comparison of valve leaflet measurements between normal and RHD (in $\mathrm{mm}$ )

\begin{tabular}{|c|c|c|c|c|c|c|}
\hline \multirow[b]{2}{*}{ Valve } & \multirow[b]{2}{*}{ Variable } & \multicolumn{2}{|c|}{ Normal $(n=288)$} & \multicolumn{2}{|c|}{ Abnormal $(n=51)$} & \multirow[b]{2}{*}{ p Value } \\
\hline & & Median & IQR & Median & IQR & \\
\hline \multirow[t]{5}{*}{ Mitral valve } & Maximal thickness AMVL tip PSLA & 2.0 & $1.7-2.4$ & 2.6 & $2.3-3.2$ & $<0.0001$ \\
\hline & Maximal thickness AMVL midpoint PSLA & 2.0 & $1.7-2.4$ & 2.8 & $2.5-3.2$ & $<0.0001$ \\
\hline & Maximal thickness PMVL midpoint & 2.2 & $1.9-2.5$ & 2.5 & $2.2-3.0$ & $<0.0001$ \\
\hline & PMVL length PSLA & 11.1 & $10.0-12.4$ & 11.2 & $10.7-12.4$ & 0.2785 \\
\hline & Maximal thickness AMVL midpoint $A 4 C$ & 2.0 & $1.8-2.4$ & 2.5 & $2.1-2.7$ & $<0.0001$ \\
\hline \multirow[t]{2}{*}{ Aortic valve } & Maximal thickness AV leaflet midpoint PSLA & 1.5 & $1.3-1.6$ & 1.9 & $1.7-2.1$ & $<0.0001$ \\
\hline & Maximal thickness AV leaflet midpoint PSSA & 1.4 & $1.2-1.6$ & 1.9 & $1.7-2.1$ & $<0.0001$ \\
\hline
\end{tabular}

AMVL, anterior mitral valve leaflet; AV, aortic valve; PMVL, posterior mitral valve leaflet; PSLA, parasternal long axis; PSSA, parasternal short axis; RHD, rheumatic heart disease. 

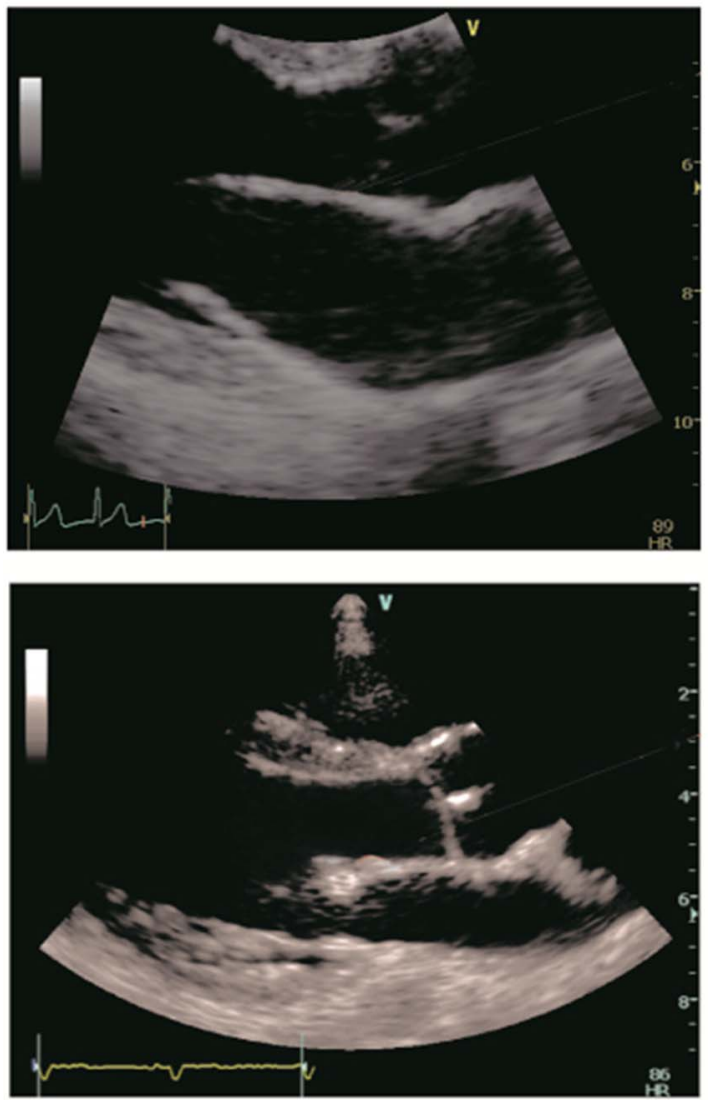

Figure 2 Zoomed two-dimensional echocardiographic view of the mitral valve, showing a normal anterior mitral valve leaflet in the upper image and a thickened anterior mitral valve leaflet tip in the lower image.

Table 2 Negative predictive value of aortic and mitral valve measurements

\begin{tabular}{lll}
\hline Measurement & NPV $(95 \%$ Cl $)$ & p Value \\
\hline AMVL tip PSLA $\leq 33 \mathrm{~mm}$ & $0.66(0.49$ to 0.84$)$ & $<0.0001$ \\
AMVL mid-PSLA $\leq 3 \mathrm{~mm}$ & $0.73(0.54$ to 0.91$)$ & $<0.0001$ \\
AV mid-PSLA $\leq 2 \mathrm{~mm}$ & $0.77(0.60$ to 0.95$)$ & $<0.0001$ \\
AV mid-PSSA $\leq 2 \mathrm{~mm}$ & $0.58(0.41$ to 0.75$)$ & $<0.0001$
\end{tabular}

AMVL, anterior mitral valve leaflet; AV, aortic valve; NPV, negative predictive valve; PSLA, parasternal long axis; PSSA, parasternal short axis.

Table 3 Positive predictive value of mitral and aortic measurements

\begin{tabular}{llc}
\hline Measurement & PPV $(95 \% \mathrm{Cl})$ & p Value \\
\hline AMVL tip PSLA $\geq 3 \mathrm{~mm}$ & $0.89(0.87$ to 0.93$)$ & $<0.0001$ \\
AMVL mid-PSLA $\geq 3 \mathrm{~mm}$ & $0.89(0.86$ to 0.93$)$ & $<0.0001$ \\
AV mid-PSLA $\geq 2 \mathrm{~mm}$ & $0.88(0.84$ to 0.92$)$ & $<0.0001$ \\
AV mid-PSSA $\geq 2 \mathrm{~mm}$ & $090(0.87$ to 0.94$)$ & $<0.0001$
\end{tabular}

AMVL, anterior mitral valve leaflet; $A V$, aortic valve; PPV, positive predictive value; PSLA, parasternal long axis; PSSA, parasternal short axis.

The results for the AMVL thickness are concordant with the limited data available from other echocardiographic and postmortem measurements of the mitral valve of normal children. The 20 normal controls of Caldas $e t a l^{7}$ had an AMVL
Table 4 Intraobserver variability

\begin{tabular}{llllll}
\hline & \multicolumn{2}{l}{ Observer 1} & & \multicolumn{2}{l}{ Observer 2} \\
\cline { 2 - 3 } \cline { 6 - 7 } & ICC & $95 \% \mathrm{Cl}$ & & ICC & $95 \% \mathrm{Cl}$ \\
\hline AMVL tip & 0.82 & 0.73 to 0.88 & & 0.90 & 0.84 to 0.93 \\
AMVL mid & 0.76 & 0.66 to 0.85 & & 0.81 & 0.73 to 0.88 \\
\hline
\end{tabular}

$\mathrm{AMVL}$, anterior mitral valve leaflet; ICC, intraclass correlation coefficient.

thickness of $1.9 \mathrm{~mm}$, but no SD of measurement was given. Atalay et $a l^{15}$ reported an indexed AMVL thickness of $1.27 \pm 0.4 \mathrm{~mm}$ in 15 healthy children aged 5-15 years. Atalay et al also reported the anterior leaflet and posterior leaflet to be thicker in children with RHD than in controls, with the result for the AMVL $(\mathrm{p}<0.001)$ more significant than for the PMVL $(\mathrm{p}<0.01)$. The postmortem study by Sahasakul et al showed that valve thickness increased with age and was not related to height, weight or body surface area (BSA). They found AMVL thickness was $1.3 \pm 0.5 \mathrm{~mm}$ in 40 subjects under 20 years of age, $1.6 \pm 0.85 \mathrm{~mm}$ in subjects $20-59$ years of age and $3.2 \pm 1.52 \mathrm{~mm}$ in subjects 60 years and older. ${ }^{19}$

The WHF echocardiographic criteria have been widely adopted since their publication, ${ }^{20-24}$ and include the $3 \mathrm{~mm}$ cut-off to define whether an anterior mitral valve is normal or thickened. A few studies have included data on mitral valve thickness. Bacquelin et al reported a $\kappa$ value of 0.6 for 2 observers measuring the AMVL in 47 children. $^{25}$ Lu et $a l^{26}$ assessed mitral valve thickness in a comparison of handheld versus portable echocardiogram platforms but raw data were not given. Ours is the first study to describe multiple parameters including PMVL normal data and supports the $3 \mathrm{~mm}$ WHF cut-off. ${ }^{20} 2526$

\section{Aortic valve}

The results showed that the median thickness of the normal aortic valve measured $1.5 \mathrm{~mm}$ in the PSLA and $1.4 \mathrm{~mm}$ in PSSA views. Note that the measurement taken in PSSA imaging is in fact a measure of the width of the edges (ie, closure lines) of two leaflets (figure 1D). Again there is minimal literature regarding the normal thickness of the aortic valve in children from either echocardiographic or pathological studies. Caldas et $a l^{7}$ reported an aortic valve thickness of $1.7 \mathrm{~mm}$ in a cohort of 20 normal children when the valve leaflets were measured in systole, presumably using PSLA imaging. The postmortem study by Sahasakul et al reported thickness at three sites on the aortic cusp increased significantly with age and was not related to height, weight or BSA. They reported a mean free edge measurement of $0.67 \mathrm{~mm} \pm 0.21 \mathrm{~mm}$ (range $0.35-1.55 \mathrm{~mm}$ ) in 40 hearts from individuals $<20$ years. ${ }^{19}$

\section{Comparisons-RHD and normal population}

Comparisons of valve thickness between normal children and children with RHD showed the AMVL to be thicker in RHD at both the AMVL tip and midpoint, and in both parasternal and apical imaging views, with $\mathrm{p}<0.0001$ for each (table 1 ).

The PMVL was also significantly thicker in children with RHD than in normal children. The PMVL length of the RHD cohort was longer than the normal cohort, which is counterintuitive to the surgical and pathological descriptions of advanced RHD. ${ }^{3} 2728$ This supports our contention that objective measurements of the PMVL are more problematic than that of the AMVL. The aortic valve was also thicker in those with RHD compared with the normal cohort regardless of whether 
the measurement was taken in PSLA or PSSA imaging. Caldas et $\mathrm{al}^{7}$ found the mitral valve to be significantly thicker in children with ARF than in normal children in a small study but did not find a difference for the aortic valve thickness.

\section{Interobserver/intraobserver variability}

Interobserver and intraobserver agreement was high for measurement of AMVL thickness. There are minimal published data in this area, particularly for normal measurements. Bacquelin et al reported $\mathrm{\kappa}$ value of 0.6 agreement for mitral valve thickness in abnormals. ${ }^{25}$ Given variability in its appearances, PMVL thickening is not used as a morphological feature, even though it is established that the PMVL thickens in advanced RHD. ${ }^{4}$

Results have been presented as discrete measurements rather than indexed to BSA as Sahasakul et al ${ }^{19}$ found that valve thickness was not related to BSA but was related to age. Moreover, measurement of height and weight are not always performed during population-based echocardiography screening programmes.

\section{Technical considerations}

Echocardiography was performed by experienced sonographers who took care to optimise image quality for valve thickness measurements. Harmonic imaging can increase clarity and the thickness of cardiac structures. ${ }^{29-31}$ The WHF criteria recommend harmonic imaging is turned off for cine-loops recorded for valve measurement. If harmonics had been inadvertently used in this study, the overall median thickness measurements would have been falsely increased, which is of lesser concern when establishing a normal cut-off.

Careful frame by frame replay is necessary in PSLA imaging to show the AMVL perpendicular to the ultrasound beam, when the AMVL is parallel to the ventricular septum. This minimises an oblique measurement of the AMVL. However, in PSLA it is not possible to be perpendicular to the PMVL, so despite the reasonable coefficient of variation, we are less confident about measures of the PMVL. We note that the measurements of the PMVL in normal children were slightly thicker than AMVL.

When measuring the tip of the AMVL, care must be taken to separate the chordal attachments from the leaflet. The interobserver agreement in this study was lower for the tip than the midpoint of the AMVL and it is recognised that the edge of the leaflet (echocardiographically the tip of the AMVL) is often the first region of the valve to develop thickening in RHD. ${ }^{32}$

\section{Clinical implications}

In normal subjects, the uppermost AMVL midpoint and AMVL tip measurements were $2.9 \mathrm{~mm}$. This is in keeping with the WHF guidelines, which recommend AMVL thickness is measured by the techniques described in this study, and classify AMVL thickening as a thickness of $\geq 3.0 \mathrm{~mm}$ for patients $\leq 20$ years. $^{4}$

We have emphasised the description of the normal findings in school-age children. The actual measurements of those with RHD will vary according to the number and severity of previous ARF episodes for that individual, the chronicity of disease and degree of mitral stenosis. ${ }^{27}$

\section{Limitations of the study}

The main objective of the study was to report the normal range of valve thickness. The RHD group comprised cases classified as RHD by WHF criteria by a panel of three cardiologists, blinded to each other's assessments. In making measurements, the reviewers were not blinded to the presence of valvular regurgitation and morphological valvular changes, which may have biased their interpretation of whether the subject had RHD or not. In other words, the observers may have subconsciously selected still frames with the 'thickest' looking valve structures. A truly blinded study would require measurers to be provided with 2D cine-loops without colour Doppler or to reduce images to single frames. The methodology used in this study reflects the practical reality of reviewing an echocardiogram.

\section{CONCLUSION}

We have described a standardised method for mitral and aortic valve measurement in children, which is objective and reproducible. Normal ranges of left heart valve thickness in a high prevalence RHD population are established. These results provide a reference range for school-age children in high prevalence RHD regions undergoing echocardiographic screening.

\section{Key messages}

What is already known about this subject?

There are no published contemporary studies describing echocardiographic measurement of mitral and aortic valve thickness in healthy children.

\section{What does this study add?}

This study provides a standardised, objective method for measurement of mitral and aortic valve thickness as well as reference ranges for normal children in high-burden acute rheumatic fever/rheumatic heart disease (RHD) populations.

How might this impact on clinical practice? Our findings support ongoing use of the 2012 World Heart Federation diagnostic criteria for RHD, which specify a $3 \mathrm{~mm}$ cut-off for mitral valve thickness.

Acknowledgements The authors would like to thank the study echocardiographers: Fiona Lean, Rachel Gatland and Sandy Long and to acknowledge Dr Tom Gentles for manuscript perusal.

Contributors RHW and NJW conceived the study. Echocardiographic measurements were performed by RHW, NC-S and NJW. Statistical analysis was performed by KS. RHW, NJW and NC-S wrote and revised the manuscript. Echocardiogram reporting according to the World Heart Federation criteria was undertaken by NJW together with Drs John Stirling, Ross Nicholson and Tom Gentles.

Funding Funding support was received from the New Zealand National Heart Foundation and the Green Lane Research and Education Fund. RHW was funded by the Joan Mary Reynolds Trust.

Competing interests None declared.

Ethics approval This study has ethical approval from the New Zealand Regional Ethics Committee: reference NTY/06/12/139.

Provenance and peer review Not commissioned; externally peer reviewed.

\section{REFERENCES}

1 van der Bel-Kahn J, Becker AE. The surgical pathology of rheumatic and floppy mitral valves. Distinctive morphologic features upon gross examination. Am J Surg Pathol 1986;10:282-92.

2 Chauvaud S, Fuzellier J-F, Berrebi A, et al. Long-term (29 years) results of reconstructive surgery in rheumatic mitral valve insufficiency. Circulation 2001;104 (Suppl 1):I-12-15. 
3 Wilkins GT, Weyman AE, Abascal VM, et al. Percutaneous balloon dilatation of the mitral valve: an analysis of echocardiographic variables related to outcome and the mechanism of dilatation. Br Heart J 1988;60:299-308.

4 Reményi $B$, Wilson $N$, Steer $A$, et al. World Heart Federation criteria for echocardiographic diagnosis of rheumatic heart disease —an evidence-based guideline. Nat Rev Cardiol 2012;9:297-309.

5 Myers PO, Tissot C, Christenson JT, et al. Aortic valve repair by cusp extension for rheumatic aortic insufficiency in children: Long-term results and impact of extension material. J Thorac Cardiovasc Surg 2010;140:836-44.

6 Bozbuga N, Erentug V, Kirali K, et al. Midterm results of aortic valve repair with the pericardial cusp extension technique in rheumatic valve disease. Ann Thorac Surg 2004:77:1272-6.

7 Caldas AM, Terreri MT, Moises VA, et al. The case for utilizing more strict quantitative Doppler echocardiographic criterions for diagnosis of subclinical rheumatic carditis. Cardiol Young 2007;17:42-7.

8 Paar JA, Berrios NM, Rose JD, et al. Prevalence of rheumatic heart disease in children and young adults in Nicaragua. Am J Cardiol 2010;105:1809-14.

9 Marijon E, Ou P, Celermajer DS, et al. Prevalence of rheumatic heart disease detected by echocardiographic screening. N Engl J Med 2007;357:470-6.

10 Carapetis JR, Hardy M, Fakakovikaetau T, et al. Evaluation of a screening protocol using auscultation and portable echocardiography to detect asymptomatic rheumatic heart disease in Tongan schoolchildren. Nat Clin Pract Cardiovasc Med 2008;5:411-17.

11 Steer AC, Kado J, Wilson N, et al. High prevalence of rheumatic heart disease by clinical and echocardiographic screening among children in Fiji. J Heart Valve Dis 2009;18:327-35; discussion 336

12 Webb RH, Wilson NJ, Lennon DR, et al. Optimising echocardiographic screening for rheumatic heart disease in New Zealand: not all valve disease is rheumatic. Cardiol Young 2011;21:436-43.

13 Sadiq M, Islam K, Abid R, et al. Prevalence of rheumatic heart disease in school children of urban Lahore. Heart 2009;95:353-7.

14 Ali SK, Eldaim IN, Osman SH, et al. Clinical and echocardiographic features of children with rheumatic heart disease and their serum cytokine profile. Pan Afr Med J 2012;13:36.

15 Atalay $\mathrm{S}, \mathrm{Uçar} \mathrm{T}, \mathrm{Ozçelik} \mathrm{N}$, et al. Echocardiographic evaluation of mitral valve in patients with pure rheumatic mitral regurgitation. Turk J Pediatr 2007;49:148-53.

16 Milne RJ, Lennon DR, Stewart JM, et al. Incidence of acute rheumatic fever in New Zealand children and youth. J Paediatr Child Health 2012;48:685-91.

17 Wilson NJ, Neutze JM. Echocardiographic diagnosis of subclinical carditis in acute rheumatic fever. Int J Cardiol 1995;50:1-6.
18 Culliford-Semmens N, Tilton E, Nicholson R, et al. PM275 Comparison of criteria for echocardiographic diagnosis of rheumatic heart disease: WHO/NIH criteria vs World Heart Federation Criteria. Global Heart 2016;11(Suppl 2):e116.

19 Sahasakul Y, Edwards WD, Naessens JM, et al. Age-related changes in aortic and mitral valve thickness: implications for two-dimensional echocardiography based on an autopsy study of 200 normal human hearts. Am J Cardiol 1988;62:424-30.

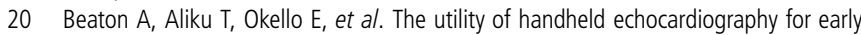
diagnosis of rheumatic heart disease. J Am Soc Echocardiogr 2014;27:42-9.

21 Rémond $M$, Atkinson $D$, White $A$, et al. Are minor echocardiographic changes associated with an increased risk of acute rheumatic fever or progression to rheumatic heart disease? Int J Cardiol 2015;198:117-22.

22 Zühlke LJ, Engel ME, Nkepu S, et al. Evaluation of a focussed protocol for hand-held echocardiography and computer-assisted auscultation in detecting latent rheumatic heart disease in scholars. Cardiol Young 2016;26:1097-106.

23 Mirabel M, Fauchier T, Bacquelin R, et al. Echocardiography screening to detect rheumatic heart disease: a cohort study of schoolchildren in French Pacific Islands. Int J Cardiol 2015;188:89-95.

24 Engelman D, Kado JH, Reményi B, et al. Focused cardiac ultrasound screening for rheumatic heart disease by briefly trained health workers: a study of diagnostic accuracy. Lancet Glob Health 2016;4:e386-94.

25 Bacquelin R, Tafflet M, Rouchon B, et al. Echocardiography-based screening for rheumatic heart disease: what does borderline mean? Int $J$ Cardiol 2016:203:1003-4.

26 Lu JC, Sable C, Ensing GJ, et al. Simplified rheumatic heart disease screening criteria for handheld echocardiography. J Am Soc Echocardiogr 2015;28:463-9.

27 Ratnakar KS, Rajagopal P, Somaraju B. Surgical pathology of mitral valves-the Indian scene. Int J Cardiol 1989;24:124-6.

28 Deshpande J, Vaideeswar P, Amonkar G, et al. Rheumatic heart disease in the past decade: an autopsy analysis. Indian Heart J 2002;54:676-80.

29 Hawkins K, Henry JS, Krasuski RA. Tissue harmonic imaging in echocardiography: better valve imaging, but at what cost? Echocardiography 2008;25:119-23.

30 Gorgulu S, Eren M, Bagirtan B, et al. Influence of different echocardiographic imaging modes on the assessment of anterior mitral leaflet thickness. J Heart Valve Dis 2005; 14:204-8.

31 Hirata K, Watanabe H, Beppu S, et al. Pitfalls of echocardiographic measurement in tissue harmonic imaging: in vitro and in vivo study. J Am Soc Echocardiogr 2002;15:1038-44.

32 Naito M, Morganroth J, Mardelli TJ, et al. Rheumatic mitral stenosis: cross-sectional echocardiographic analysis. Am Heart J 1980;100:34-40. 